\title{
Clinical Translation of Cerebral Preconditioning
}

\author{
Guohua Xi
}

Published online: 23 February 2010

(C) Springer Science+Business Media, LLC 2010

Cerebral preconditioning (PC) refers to a phenomenon whereby prior treatment with a variety of stimuli can induce protection against later brain injury $[1,2]$. It is well known that PC is one of the most powerful methods of reducing stroke-induced brain injury in animal models. Preconditioning has been used for heart and brain surgery and is now a clinical reality $[3,4]$. In the brain, ischemic PC has been used during clipping of cerebral aneurysm and attenuates tissue hypoxia during aneurysm clipping [5].

A preconditioning workshop was held at the University of Michigan in Ann Arbor on July 24 to 25, 2009. The workshop brought together 20 experts from the United States who discussed the mechanisms of PC-induced neuroprotection and how to translate those results to the clinic.

Neuroprotection is a major issue in neurosurgery since neurosurgical procedures may result in trauma, hemorrhage, and/or ischemia. Cognitive dysfunction and ischemic brain damage can also occur in patients after heart bypass surgery and carotid endarterectomy. Dr. Michael Wang (University of Michigan) reviewed clinical targets of PC and suggested that brain injury after neurosurgery, carotid endarterectomy and coronary artery bypass graft surgery, and vasospasm following subarachnoid hemorrhage are major potential clinical targets for cerebral PC [6].

Mechanisms of PC were updated during the workshop. Dr. John Hallenbeck (National Institute of Neurological Disorders and Stroke) presented natural tolerance in hibernation torpor as a guide to target selection for stroke and his recent findings indicate that SUMOylation has an

G. Xi (₫)

Department of Neurosurgery, University of Michigan,

Ann Arbor, MI 48109-2200, USA

e-mail: guohuaxi@umich.edu important role in ischemic tolerance [7]. Whether or not cerebral blood flow has a role in PC-induced neuroprotection was presented by Dr. Thaddeus Nowak (University of Tennessee). Dr. Anne Stetler (University of Pittsburgh) reviewed molecular mechanisms of PC [8]. Although results from animal studies are promising, most PC studies have been done in young and healthy animals. Whether or not aged, hypertensive, or diabetic animals should be included in future preconditioning experiments was also discussed. In addition, data showing that inducible nitrous oxide synthase promoter polymorphism affords protection against cognitive dysfunction after carotid endarterectomy was reported by Dr. Raqeeb Haque (Columbia University) [9], and the results suggest that cognitive dysfunction could be an endpoint to measure success in future potential preconditioning trials.

To develop safe and noninvasive PC approaches is critical for clinical translation of cerebral PC. Recently, many noninvasive PC methods are available to induce brain tolerance [2]. Dr. Jeffrey Gidday (Washington University) summarized pharmacologic PC. There are many promising approaches, such as PC with anesthetics, deferoxamine and erythropoietin [10]. Dr. John Zhang (Loma Linda University) reviewed data on hyperbaric oxygen PC and demonstrated that this form of PC can reduce traumatic, ischemic, and hemorrhagic brain injury $[11,12]$. Dr. Miguel PerezPinzon (University of Miami) presented that pretreatment with resveratrol, a natural polyphenol found in grapes and wine, reduces ischemic brain injury [13, 14].

Preconditioning is induced by stimuli which can induce brain tolerance but not cell injury, but safety is a major concern because there are no boundaries between PCinduced brain tolerance and cell death [15]. It is well known that many effective treatments for stroke require pretreatment. Therefore, Dr. Richard Keep (University of Michigan) 
discussed prophylactic treatment vs. preconditioning for reducing surgery-related complications [6]. Recent experiments also suggest that postconditioning can reduce ischemic injury in heart and brain. Dr. Heng Zhao emphasized postconditioning-induced neuroprotection and discussed the potential for clinical translation [16].

Lastly, Dr. Thomas Kent (Baylor University) and Dr. Sebastian Koch (University of Miami) led a session to discuss the feasibility of using PC in humans. Many issues were discussed. For example, is there enough preclinical evidence to proceed? Can humans be preconditioned? What is the most appropriate clinical setting? What are the next steps [6]?

The two-day workshop was ended successfully and enhanced our understanding of cerebral PC. The next PC workshop has been planned in Miami (organizer: Dr. Miguel Perez-Pinzon). It is time to translate the concept of $\mathrm{PC}$ to the clinic.

\section{References}

1. Gidday JM (2006) Cerebral preconditioning and ischaemic tolerance. Nat Rev Neurosci 7(6):437-448

2. Dirnagl U, Becker K, Meisel A (2009) Preconditioning and tolerance against cerebral ischaemia: from experimental strategies to clinical use. Lancet Neurol 8(4):398-412

3. Yellon DM, Dana A (2000) The preconditioning phenomenon: a tool for the scientist or a clinical reality? Circ Res 87:543-550

4. Steiger HJ, Hanggi D (2007) Ischaemic preconditioning of the brain, mechanisms and applications. Acta Neurochirurgica 149 (1): $1-10$
5. Chan MT, Boet R, Ng SC, Poon WS, Gin T (2005) Effect of ischemic preconditioning on brain tissue gases and $\mathrm{pH}$ during temporary cerebral artery occlusion. Acta Neurochir Suppl 95:93-96

6. Keep RF, Wang MM, Xiang J, Hua Y, Xi G (2010) Is there a place for cerebral preconditioning in the clinic? Translational Stroke Research. doi:10.1007/s12975-009-0007-7

7. Lee Y, Castri P, Bembry J, Maric D, Auh S, Hallenbeck JM (2009) SUMOylation participates in induction of ischemic tolerance. J Neurochem 109(1):257-267

8. Stetler RA, Zhang F, Liu C, Chen J (2008) Ischemic tolerance as an active and intrinsic neuroprotective mechanism. Handb Clin Neurol 92:171-195

9. Yocum GT, Gaudet JG, Lee SS, Stern Y, Teverbaugh LA, Sciacca RR et al (2009) Inducible nitric oxide synthase promoter polymorphism affords protection against cognitive dysfunction after carotid endarterectomy. Stroke 40(5):1597-1603

10. Gidday JM (2010) Pharmacologic preconditioning: translating the promise. Translational Stroke Research. doi:10.1007/s12975-0100011-y

11. Qin Z, Song S, Xi G, Silbergleit R, Keep RF, Hoff JT et al (2007) Preconditioning with hyperbaric oxygen attenuates brain edema after experimental intracerebral hemorrhage. Neurosurg Focus 22(5):E13

12. Jadhav V, Ostrowski RP, Tong W, Matus B, Jesunathadas R, Zhang JH (2009) Cyclo-oxygenase-2 mediates hyperbaric oxygen preconditioning-induced neuroprotection in the mouse model of surgical brain injury. Stroke 40(9):3139-3142

13. Della-Morte D, Dave KR, DeFazio RA, Bao YC, Raval AP, Perez-Pinzon MA (2009) Resveratrol pretreatment protects rat brain from cerebral ischemic damage via a sirtuin 1-uncoupling protein 2 pathway. Neuroscience 159(3):993-1002

14. Kim EJ, Raval AP, Hirsch N, Perez-Pinzon MA (2010) Ischemic preconditioning mediates cyclooxygenase-2 expression via nuclear factor-kappa B activation in mixed cortical neuronal cultures. Translational Stroke Research. doi:10.1007/s12975-009-0006-8

15. Dirnagl U, Simon RP, Hallenbeck JM (2003) Ischemic tolerance and endogenous neuroprotection. Trends Neurosci 26(5):248-254

16. Zhao H (2009) Ischemic postconditioning as a novel avenue to protect against brain injury after stroke. J Cereb Blood Flow Metab 29(5):873-885 